\title{
Ru-Catalyzed Selective C-H Functionalization of Pyridotriazoles with Acrylates
}

\author{
Abhisek Joshi \\ Rashmi Semwal \\ Subbarayappa Adimurthy* (D) \\ Academy of Scientific \& Innovative Research, Ghaziabad; CSIR- \\ Central Salt \& Marine Chemicals Research Institute, G. B. Marg, \\ Bhavnagar-364 002, Gujarat, India \\ adimurthy@csmcri.res.in
}

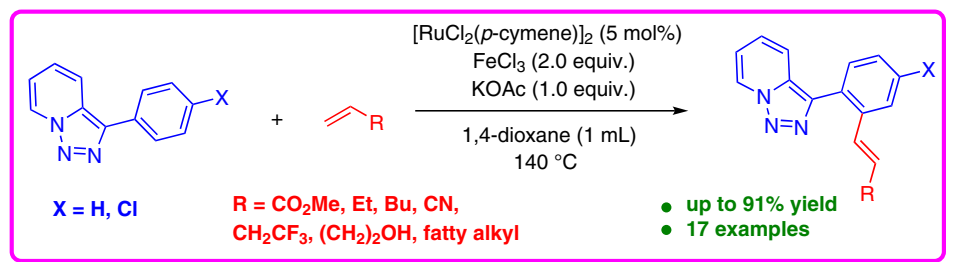

Received: 23.09 .2021

Accepted after revision: 04.10.2021

Published online: 04.10.2021

DOI: 10.1055/a-1661-5655; Art ID: so-2021-d0047-I

License terms: $c(9)$

(c) 2021. The Author(s). This is an open access article published by Thieme under the terms of the Creative Commons Attribution-NonDerivative-NonCommercial-License, permitting copying and reproduction so long as the original work is given appropriate credit. Contents may not be used for commercial purposes or adapted, remixed, transformed or built upon. (https://creativecommons.org/licenses/by-nc-nd/4.0/)

Abstract Ruthenium-catalyzed efficient and selective $\mathrm{C}-\mathrm{H}$ alkenylation of pyridotriazoles with acrylates is described. The combination of metals (Ru and Fe) plays a crucial role in achieving quantitative yields of the desired products. The reaction is proposed to involve the formation of a ruthenium cyclometalated intermediate.

Key words $\mathrm{C}-\mathrm{H}$ functionalization, pyridotriazoles, acrylates

Transition-metal-catalyzed $\mathrm{C}-\mathrm{H}$ bond activation has emerged as a new tool in organic synthesis for the functionalization of arenes. ${ }^{1}$ In general, selective $\mathrm{C}-\mathrm{H}$ functionalization is controlled by the directing/functional groups present on the substrates. Therefore, the use of directing groups by transition-metal-catalyzed $\mathrm{C}-\mathrm{H}$ functionalization has aroused much attention in recent years. ${ }^{2}$ Directing groups such as carboxyl, ${ }^{3}$ carbonyl, ${ }^{4}$ cyano, ${ }^{5}$ and hydroxyl $l^{6}$ are wellknown for various transition-metal-catalyzed $\mathrm{C}-\mathrm{H}$ functionalizations of arenes.

Pyridotriazole is an important scaffold in organic chemistry and plays an important role in several transition-metal-catalyzed denitrogenative transannelation reactions to generate diverse heterocycles. ${ }^{7,8}$ However, to the best of our knowledge, $\mathrm{C}-\mathrm{H}$ alkenylation reaction using the pyridotriazole as a directing group to form $\mathrm{C}\left(\mathrm{sp}^{2}\right)-\mathrm{C}\left(\mathrm{sp}^{2}\right)$ bonds has never been attempted. In continuation of our works on the transannelation of pyridotriazoles, ${ }^{8}$ we herein describe the ruthenium-catalyzed regioselective alkenylation of pyridotriazoles (Scheme 1).

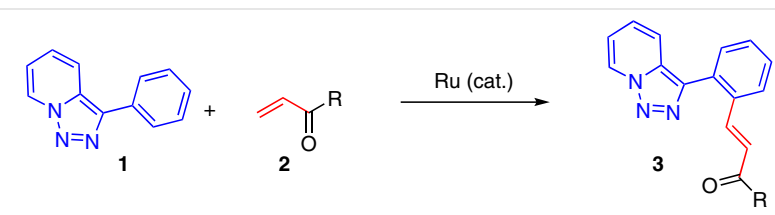

Scheme 1 Alkenylation of pyridotriazoles

Initially, we focused on the optimization of reaction conditions for the selective alkenylation of pyridotriazole 1a with $\mathbf{2 a}$ in the presence of $\left[\mathrm{RuCl}_{2}(p \text {-cymene })\right]_{2}$ (5 mol\%) as the catalyst with 2.0 equiv. of potassium acetate at $140{ }^{\circ} \mathrm{C}$ in 1,4-dioxane $(1 \mathrm{~mL})$ for $36 \mathrm{~h}$ (Table 1$)$. Under these conditions, a trace amount of product 3a formation was observed (entry 1 ). With $\mathrm{Cu}(\mathrm{OAc})_{2}$ (0.5 equiv.) as an additive in the absence of base, $20 \%$ yield of 3a was obtained (entry 2 ).

When the reaction was performed without any catalyst, no product formation was observed (entry 3 ). When the reaction was performed with $5 \mathrm{~mol} \%\left[\mathrm{RuCl}_{2}(p \text {-cymene })\right]_{2}, 0.5$ equiv. of $\mathrm{Cu}(\mathrm{OAc})_{2}$, and 2.0 equiv. of $\mathrm{KOAc}$, a $42 \%$ yield of $\mathbf{3 a}$ was obtained after $48 \mathrm{~h}$ (entry 4). After screening various solvents (DCE, toluene, DMF, and DMSO) as well as different catalysts ( $\mathrm{Co}, \mathrm{Ni}$, and $\mathrm{Pd}$ ) either no reaction or no improvement in yield was observed (entries 5-12). On increasing the amount of additive $\mathrm{Cu}(\mathrm{OAc})_{2}$, to one and two equivalents, the yield of the product was increased to $62 \%$ (entries 13 and 14). Performing the reaction with two equivalents of $\mathrm{ZnCl}_{2}$ and $\mathrm{FeCl}_{3}$ as additives $36 \%$ and $73 \%$ yields of desired product were obtained, respectively (entries 15 and 16). With $\mathrm{FeCl}_{3}$ as additive, the amount of base (KOAc) was decreased to 1.0 equiv., and under these conditions the desired product 3a was obtained in $90 \%$ yield (entry 17 ). On decreasing the base to 0.5 equiv. the yield was reduced to $74 \%$ (entry 18). The best yield of 3a was thus obtained un- 
Table 1 Optimization of Reaction Conditions for $3 a^{a}$

\begin{tabular}{|c|c|c|c|c|c|}
\hline Entry & $\begin{array}{l}\text { Catalyst } \\
\text { (5 mol\%) }\end{array}$ & $\begin{array}{l}\text { Additive } \\
\text { (equiv.) }\end{array}$ & $\begin{array}{l}\text { Base } \\
\text { (equiv.) }\end{array}$ & $\begin{array}{l}\text { Solvent } \\
(1 \mathrm{~mL})\end{array}$ & Yield (\%) \\
\hline 1 & {$\left[\mathrm{RuCl}_{2}(p \text {-cymene })\right]_{2}$} & - & $\operatorname{KOAC}(2.0)$ & 1,4-dioxane & trace \\
\hline 2 & {$\left[\mathrm{RuCl}_{2}(p \text {-cymene })\right]_{2}$} & $\mathrm{Cu}(\mathrm{OAc})_{2}(0.5)$ & - & 1,4-dioxane & 20 \\
\hline 3 & - & $\mathrm{Cu}(\mathrm{OAc})_{2}(0.5)$ & $\operatorname{KOAC}(2.0)$ & 1,4-dioxane & $\mathrm{nr}$ \\
\hline 4 & {$\left[\mathrm{RuCl}_{2}(p \text {-cymene })\right]_{2}$} & $\mathrm{Cu}(\mathrm{OAc})_{2}(0.5)$ & $\operatorname{KOAC}(2.0)$ & 1,4-dioxane & 42 \\
\hline 5 & {$\left[\mathrm{RuCl}_{2}(p \text {-cymene })\right]_{2}$} & $\mathrm{Cu}(\mathrm{OAc})_{2}(0.5)$ & $\operatorname{KOAC}(2.0)$ & DCE & $\mathrm{nr}$ \\
\hline 6 & {$\left[\mathrm{RuCl}_{2}(p \text {-cymene })\right]_{2}$} & $\mathrm{Cu}(\mathrm{OAc})_{2}(0.5)$ & $\operatorname{KOAC}(2.0)$ & toluene & $\mathrm{nr}$ \\
\hline 7 & {$\left[\operatorname{RuCl}_{2}(p \text {-cymene })\right]_{2}$} & $\mathrm{Cu}(\mathrm{OAc})_{2}(0.5)$ & $\operatorname{KOAC}(2.0)$ & DMF & $\mathrm{nr}$ \\
\hline 8 & {$\left[\mathrm{RuCl}_{2}(p \text {-cymene })\right]_{2}$} & $\mathrm{Cu}(\mathrm{OAc})_{2}(0.5)$ & $\operatorname{KOAC}(2.0)$ & DMSO & $\mathrm{nr}$ \\
\hline 9 & $\mathrm{Pd}(\mathrm{OAc})_{2}$ & $\mathrm{Cu}(\mathrm{OAc})_{2}(0.5)$ & $\operatorname{KOAC}(2.0)$ & 1,4-dioxane & 26 \\
\hline 10 & $\mathrm{Pdl}_{2}$ & $\mathrm{Cu}(\mathrm{OAc})_{2}(0.5)$ & $\operatorname{KOAC}(2.0)$ & 1,4-dioxane & 20 \\
\hline 11 & $\mathrm{Co}(\mathrm{OAc})_{2}$ & $\mathrm{Cu}(\mathrm{OAc})_{2}(0.5)$ & $\operatorname{KOAC}(2.0)$ & 1,4-dioxane & $\mathrm{nr}$ \\
\hline 12 & $\mathrm{Ni}(\mathrm{acac})_{2}$ & $\mathrm{Cu}(\mathrm{OAc})_{2}(0.5)$ & $\operatorname{KOAC}(2.0)$ & 1,4-dioxane & $\mathrm{nr}$ \\
\hline 13 & {$\left[\operatorname{RuCl}_{2}(p \text {-cymene })\right]_{2}$} & $\mathrm{Cu}(\mathrm{OAc})_{2}(1.0)$ & $\operatorname{KOAC}(2.0)$ & 1,4-dioxane & 53 \\
\hline 14 & {$\left[\mathrm{RuCl}_{2}(p \text {-cymene })\right]_{2}$} & $\mathrm{Cu}(\mathrm{OAc})_{2}(2.0)$ & $\operatorname{KOAC}(2.0)$ & 1,4-dioxane & 62 \\
\hline 15 & {$\left[\mathrm{RuCl}_{2}(p \text {-cymene })\right]_{2}$} & $\mathrm{ZnCl}_{2}(2.0)$ & $\operatorname{KOAC}(2.0)$ & 1,4-dioxane & 36 \\
\hline 16 & {$\left[\operatorname{RuCl}_{2}(p \text {-cymene })\right]_{2}$} & $\mathrm{FeCl}_{3}(2.0)$ & $\operatorname{KOAC}(2.0)$ & 1,4-dioxane & 73 \\
\hline 17 & $[$ RuCl 2 (p-cymene $)]_{2}$ & $\mathrm{FeCl}_{3}(2.0)$ & $\operatorname{KOAC}(1.0)$ & 1,4-dioxane & 90 \\
\hline 18 & {$\left[\operatorname{RuCl}_{2}(p \text {-cymene })\right]_{2}$} & $\mathrm{FeCl}_{3}(2.0)$ & $\operatorname{KOAC}(0.5)$ & 1,4-dioxane & 74 \\
\hline
\end{tabular}

${ }^{a}$ Conditions: $1 \mathbf{a}(0.25 \mathrm{mmol}), \mathbf{2 a}(0.5 \mathrm{mmol})$, catalyst, additive, base, solvent $(1 \mathrm{~mL})$, in an oil bath at $140{ }^{\circ} \mathrm{C}$ for $24 \mathrm{~h}$.

$\mathrm{b}$ Isolated yield.

der the conditions of entry 17; hence these parameters were set as optimum for further alkenylations of pyridotriazoles with different acrylates (Table 1 ).

With this set of optimized conditions, the $\mathrm{C}-\mathrm{H}$ functionalization of 3-phenyl[1,2,3]triazolo[1,5-a]pyridine (1a) with different acrylates was examined (Scheme 2).

Acrylates bearing Me, Et, and Bu groups at the terminal position reacted smoothly with 3-phenyl-[1,2,3]triazolo[1,5-a]pyridine (1a) to afford the corresponding alkenylated products 3a-c in good to excellent yields (72-90\%). The reaction of methyl methacrylate also gave the corresponding functionalized product 3d in good yield (87\%). Other acrylates such as 2-methoxyethyl acrylate, 2-hydroxyethyl (Z)-but-2-enoate, and 2,2,2-trifluoroethyl acrylate reacted well under the optimized conditions and afforded the corresponding products $\mathbf{3 e -} \mathbf{g}$ in good to excellent yields (70-90\%). Under the same conditions, 3[(allyloxy)methyl]heptane and acrylonitrile afforded the corresponding products $\mathbf{3 h}$ and $\mathbf{3 i}$ in $91 \%$ and $67 \%$ yields, respectively. As is evident from the yields of products $\mathbf{3 f}-\mathbf{i}$, ef- fects associated with electron-donating or electron-withdrawing substituents on the acrylate moiety do not affect the efficiency of the transformation.

This transformation is not limited to the 3-phenyl $[1,2,3]$ triazolo[1,5-a]pyridine (1a); indeed, the triazole bearing a chlorine substituent at the 4-position of the phenyl ring 3-(4-chlorophenyl)-[1,2,3]triazolo[1,5-a]pyridine (1b) proved to be amenable to this procedure under the same optimized conditions. Similar reactivities of a range of acrylates were observed with $\mathbf{1 b}$ and afford the differently functionalized products $\mathbf{4 a - h}$ in good yields ranging from 65-88\% (Scheme 3).

To gain insight into the reaction mechanism, some control experiments were performed (Scheme 4). Initially, the reaction was conducted by the addition of the radical scavenger TEMPO under the optimized conditions to establish whether the reaction proceeds via a radical or ionic pathway (Scheme 4a). Under these conditions, no adduct formation was observed, indicating that the reaction does not proceed through a radical pathway. However, 2-benzoyl pyridine (5) was observed as a side product. To establish 

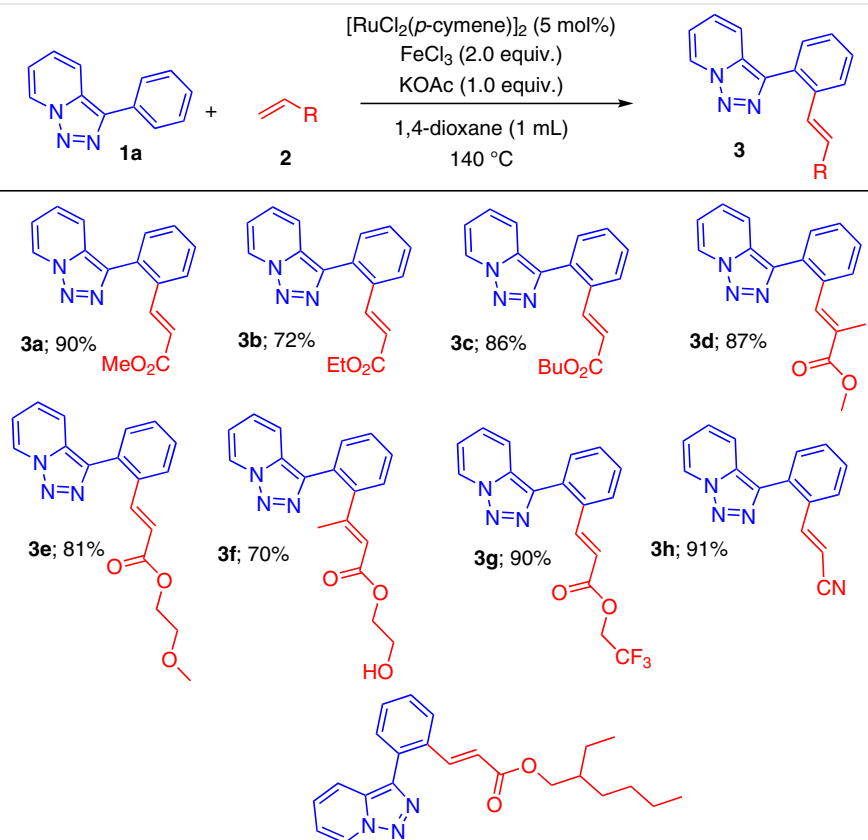

3i; $67 \%$

Scheme 2 Substrate scope of triazolo[1,5-a]pyridines. Reagents and conditions: 1 a (0.25 mmol), 2 ( 0.5 mmol), catalyst (5 mol\%), additive (2.0 equiv.), base (1.0 equiv.), solvent ( $1 \mathrm{~mL})$, in an oil bath, isolated yield.
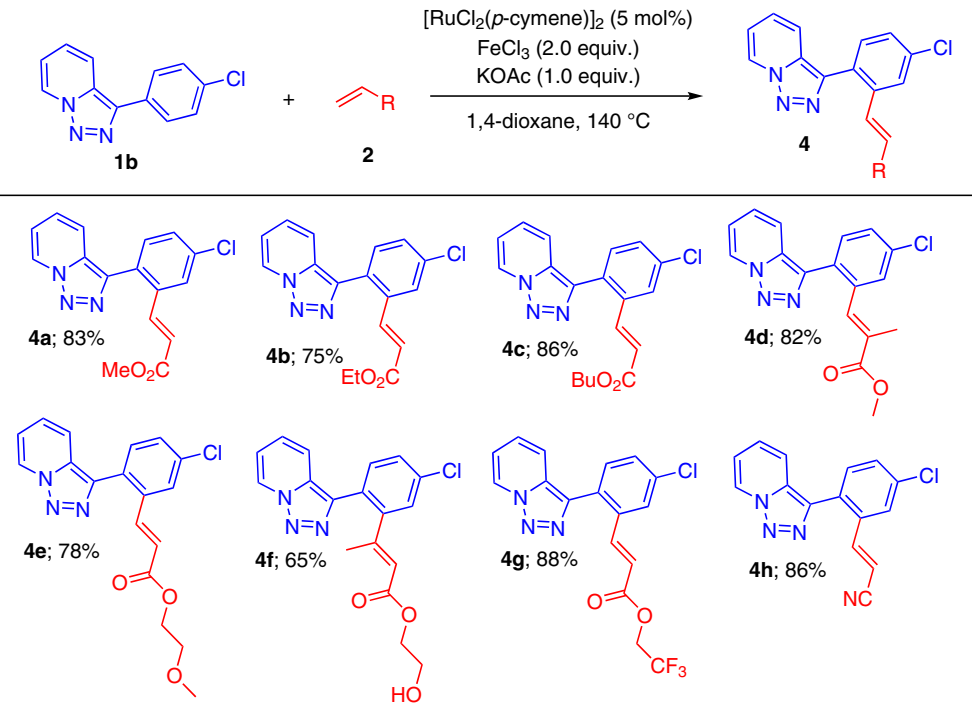

Scheme 3 Substrate scope with 3-(4-chlorophenyl)-[1,2,3]triazolo[1,5-a]pyridine. Reagents and conditions: 1 b (0.25 mmol), 2 (0.5 mmol), catalyst (5 mol\%), additive (2.0 equiv.), base (1.0 equiv.), solvent (1 mL), in carousel reaction station, $48 \mathrm{~h}$, isolated yield.

possible intermediates, 2-benzylpyridine $(\mathbf{6})$ was reacted with 2a under the standard conditions, but the expected product 3a was not observed (Scheme 4b). Furthermore, when 2-benzoylpyridine (5) was reacted with $\mathbf{2 a}$ under the same conditions, it did not yield $\mathbf{3 a}$ (Scheme 4c). These two reactions (Scheme $4 \mathrm{~b}$ and c) suggest that both $\mathbf{5}$ and $\mathbf{6}$ are not intermediates in the reaction pathway. 


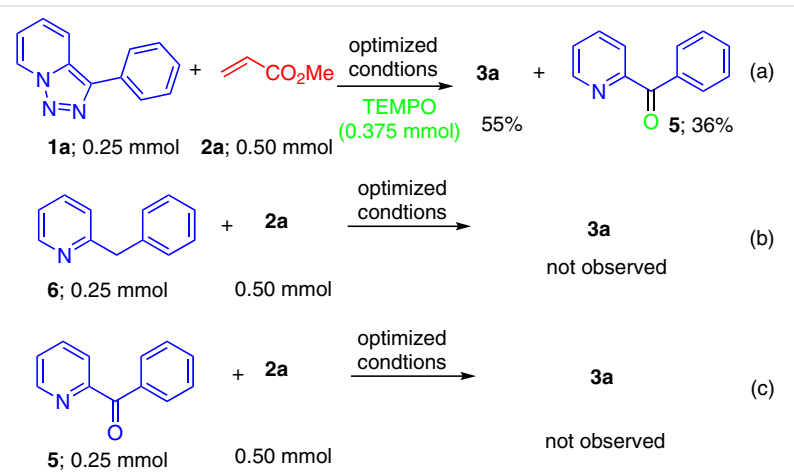

Scheme 4 Control experiments

Based on the control experiments and literature reports, ${ }^{9}$ a plausible reaction mechanism is proposed (Scheme 5). Initially, $\left[\mathrm{RuCl}_{2} \text { (p-cymene) }\right]_{2}$ in the presence of base (KOAc) generates the active $\mathrm{Ru}(\mathrm{II})$ species $\mathbf{A}$, which upon coordination with a nitrogen of the pyridotriazole ring and subsequent ligand-assisted $\mathrm{C}-\mathrm{H}$ ruthenation via intermediate $\mathbf{B}$ gives the ruthenacyclic intermediate $\mathbf{C}$.

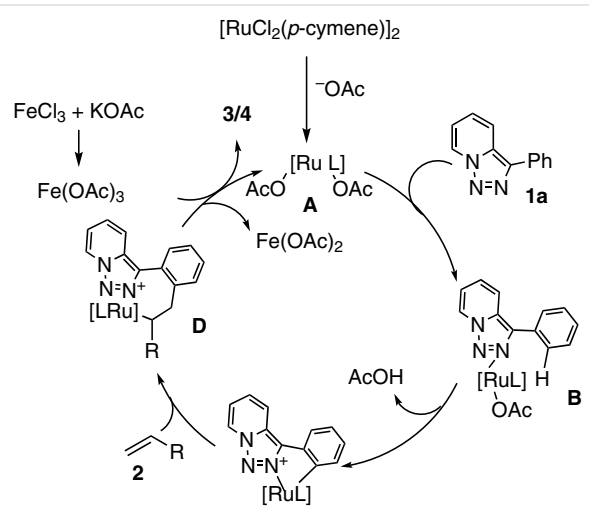

C

Scheme 5 Plausible reaction mechanism

Coordination of the Ru center of $\mathbf{C}$ with the addition of the alkene leads to the ruthenacyclic intermediate $\mathbf{D}$ that, followed by $\beta$-hydride elimination, gives the desired alkenylated products $\mathbf{3} / \mathbf{4}$.

In conclusion, we have developed a ruthenium-catalyzed regioselective $\mathrm{C}-\mathrm{H}$ alkenylation of pyridotriazoles with a range of acrylates. ${ }^{10}$ Different acrylates bearing Me, Et, Bu, trifluoroethyl, 2-methoxyethyl acrylate, 2-hydroxyethyl (Z)-but-2-enoate, 3-((allyloxy)methyl)heptane, and acrylonitrile reacted smoothly and afforded the corresponding products in good yields. Control experiments suggest that the reaction proceeds through an ionic pathway.

\section{Conflict of Interest}

The authors declare no conflict of interest.

\section{Funding Information}

S. R. is grateful to the Department of Science and Technology, Ministry of Science and Technology, India (DST) for the award of an INSPIRE Fellowship. We also thank CSIR-CSMCRI (Grant Numbers MLP-0027 and MLP-320) for partial financial support.

\section{Acknowledgment}

CSIR-CSMCRI communication No. 184/2021. S. R. is grateful to AcSIR for their PhD enrolment scheme and the 'Analytical Discipline and Centralized Instrumental Facilities' for providing instrumentation facilities.

\section{Supporting Information}

Supporting information for this article is available online at https://doi.org/10.1055/a-1661-5655.

\section{References and Notes}

(1) For reviews, see: (a) Kalepu, J. Pilarski L. T. Molecules 2019, 24, 830. (b) Mihai, M. T.; Genov, G. R.; Phipps, R. J. Chem. Soc. Rev. 2018, 47, 149. (c) Sambiagio, C.; Schönbauer, D.; Blieck, R.; DaoHuy, T.; Pototschnig, G.; Schaaf, P.; Wiesinger, T.; Zia, M. F. F.; Wencel-Delord, J.; Besset, T.; Maes, B. U. W. Schnürch M. Chem. Soc. Rev. 2018, 47, 6603. (d) Xu, Y. Y.; Dong, G. Chem. Sci. 2018, 9, 1424. (e) Zhang, Z.; Dixneuf, P. H.; Soule, J.-F. Chem. Commun. 2018, 54, 7265. (f) da Silva, E. N. Jr.; Jardim, G. A. M.; Gomes, R. S.; Liang, Y.-F.; Ackermann, L. Chem. Commun. 2018, 54, 7398. (g) Shang, M.; Sun, S.-Z.; Wang, H.-L.; Wang, M.-M.; Dai, H.-X. Synthesis 2016, 48, 4381. (h) Molnár, Á.; Papp, A. Curr. Org. Chem. 2016, 20, 381. (i) Chen, Z.; Wang, B.; Zhang, J.; Yu, W.; Liu, Z.; Zhang, Y. Org. Chem. Front. 2015, 2, 1107. (j) Yuan, K.; Soulé, J.-F.; Doucet, H. ACS Catal. 2015, 5, 978. (k) Girard, S. A.; Knauber, T.; Li, C.-J. Angew. Chem. Int. Ed. 2014, 53, 74. (l) Zhou, L.; Lu, W. Chem. Eur. J. 2014, 20, 634. (m) Kuhl, N.; Hopkinson, M. N.; Wencel-Delord, J.; Glorius, F. Angew. Chem. Int. Ed. 2012, 51, 10236. (n) Cho, S. H.; Kim, J. Y.; Kwak, J.; Chang, S. Chem. Soc. Rev. 2011, 40, 5068. (o) Ackermann, L.; Vicente, R.; Kapdi, A. R. Angew. Chem. Int. Ed. 2009, 48, 9792.

(2) (a) Tan, G.; You, Q.; You, J. ACS Catal. 2018, 8, 8709. (b) Kulkarni, A. A.; Daugulis, O. Synthesis 2009, 4087. (c) Gao, K.; Yoshikai, N. Acc. Chem. Res. 2014, 47, 1208. (d) Liu, W.; Ackermann, L. ACS Catal. 2016, 6, 3743.

(3) (a) Giri, R.; Maugel, N.; Li, J.-J.; Wang, D.-H.; Breazzano, S. P.; Saunders, L. B.; Yu, J.-Q. J. Am. Chem. Soc. 2007, 129, 3510. (b) Giri, R.; Yu, J.-Q. J. Am. Chem. Soc. 2008, 130, 14082. (c) Wang, D.-H.; Mei, T.-S.; Yu, J.-Q. J. Am. Chem. Soc. 2008, 130, 17676. (d) Zhang, Y.-H.; Yu, J.-Q. J. Am. Chem. Soc. 2009, 131, 14654. (e) Wang, D.-H.; Engle, K. M.; Shi, B.-F.; Yu, J.-Q. Science 2010, 327, 315. (f) Engle, K. M.; Wang, D.-H.; Yu, J.-Q. Angew. Chem. Int. Ed. 2010, 49, 6169.

(4) (a) Gandeepan, P.; Parthasarathy, K.; Cheng, C. H. J. Am. Chem. Soc. 2010, 132, 8569. (b) Patureau, F. W.; Besset, T.; Glorius, F. Angew. Chem. Int. Ed. 2011, 50, 1064. 
(5) (a) Leow, D.; Li, G.; Mei, T.-S.; Yu, J.-Q. Nature 2012, 486, 518. (b) Li, W.; Xu, Z. P.; Sun, P. P.; Jiang, X. Q.; Fang, M. Org. Lett. 2011, 13, 1286. (c) Li, W.; Sun, P. P. J. Org. Chem. 2012, 77, 8362. (d) Du, B. N.; Jiang, X. Q.; Sun, P. P. J. Org. Chem. 2013, 78, 2786.

(6) Zhang, C.; Ji, J.; Sun, P. J. Org. Chem. 2014, 79, 3200.

(7) (a) Chuprakov, S.; Hwang, F.; Gevorgyan, W. V. Angew. Chem. Int. Ed. 2007, 46, 4757. (b) Helan, V.; Gulevich, A. V.; Gevorgyan, V. Chem. Sci. 2015, 6, 1928. (c) Shi, Y.; Gevorgyan, V. Chem. Commun. 2015, 51, 17166. (d) Yadagiri, D.; Anbarasan, A. Org. Lett. 2014, 16, 2510. (e) Shin, S.; Park, Y.; Kim, C. E.; Son, J. Y.; Lee, P. H. J. Org. Chem. 2015, 80, 5859. (f) Zhang, Z.; Gevorgyan, V. Org. Lett. 2020, 22, 8500.

(8) (a) Joshi, A.; Chandra Mohan, D.; Adimurthy, S. Org. Lett. 2016, 18, 464. (b) Joshi, A.; Chandra Mohan, D.; Adimurthy, S. J. Org. Chem. 2016, 81, 9461. (c) Rawat, D.; Ravi, C.; Joshi, A.; Suresh, E.; Jana, K.; Ganguly, B.; Adimurthy, S. Org. Lett. 2019, 21, 2043. (d) Joshi, A.; Semwal, R.; Suresh, E.; Adimurthy, S. Chem. Commun. 2019, 55, 10888. (e) Rawat, D.; Kumar, R.; Adimurthy, S. Eur. J. Org. Chem. 2019, 7874.

(9) Pipaliya, B. V.; Seth, K.; Chakraborti, A. K. Chem. Asian J. 2021, $16,87$.

\section{(10) Experimental Section}

All commercially available chemicals and reagents were used without any further purification unless otherwise indicated. ${ }^{1} \mathrm{H}$ and ${ }^{13} \mathrm{C}$ NMR spectra were recorded at $600,200,150$, and 125 $\mathrm{MHz}$, respectively. Spectra were recorded in $\mathrm{CDCl}_{3}$ as solvent. Multiplicity is indicated as follows: $\mathrm{s}$ (singlet), $\mathrm{d}$ (doublet), $\mathrm{t}$ (triplet), m (multiplet), dd (doublet of doublets), and coupling constants $(J)$ are given in Hz. Chemical shifts are reported in ppm relative to TMS as an internal standard. Mass spectra were obtained using electron impact (EI) ionization. Progress of the reactions was monitored by thin-layer chromatography (TLC). All products were purified through column chromatography using silica gel with 100-200 mesh size using ethyl acetate/hexane as eluent unless otherwise stated.

General Procedures(A) Synthesis of Triazolopyridine Derivatives 1

Hydrazine monohydrate $(0.30 \mathrm{mmol})$ and acetic acid $(0.02$ $\mathrm{mmol}$ ) were added to a solution of the requisite 2-acylpyridine $(0.20 \mathrm{mmol})$ in ethanol $(1.0 \mathrm{~mL})$ at room temperature. The reaction mixture was heated at reflux for $6 \mathrm{~h}$, and then EtOAc (5.0 $\mathrm{mL})$ and $\mathrm{Cu}(\mathrm{OAc})_{2}(0.01 \mathrm{mmol})$ were added. After stirring at the indicated temperature for the indicated time, the resulting mixture was cooled to room temperature and then diluted with EtOAc $(20 \mathrm{~mL})$. The organic phase was washed with water (10 $\mathrm{mL}$ ) and then dried over anhydrous $\mathrm{Na}_{2} \mathrm{SO}_{4}$. After filtration and concentration under reduced pressure, followed by purification by column chromatography, the desired triazolopyridine derivatives were isolated.

(B) Synthesis of Methyl (E)-3-(2-\{[1,2,3]Triazolo[1,5-a]pyridin-3-yl\}phenyl)acrylate (3a)

To a reaction tube equipped with a magnetic stir bar, 3-phenyl[1,2,3]triazolo[1,5-a]pyridine (1a, $48.8 \mathrm{mg}, 0.25 \mathrm{mmol}$ ), methyl acrylate (2a, $43.1 \mathrm{mg}, 0.5 \mathrm{mmol}),\left[\mathrm{RuCl}_{2}(p \text {-cymene) }]_{2}\right.$ (6.75 mg, $5 \mathrm{~mol} \%), \mathrm{FeCl}_{3}(81.1 \mathrm{mg}, 0.5 \mathrm{mmol})$, and base KOAc (24.5 mg, $0.25 \mathrm{mmol}$ ) were added, followed by dry 1,4-dioxane $(1 \mathrm{~mL})$. The mixture was heated in a carousel reaction station at $140{ }^{\circ} \mathrm{C}$ in a closed tube for $48 \mathrm{~h}$, and progress was monitored by TLC. After completion of reaction, it was allowed to cool to room temperature. Then the mixture was poured into brine $(30 \mathrm{~mL})$.
The product was extracted with EtOAc $(3 \times 15 \mathrm{~mL})$ and dried with anhydrous $\mathrm{Na}_{2} \mathrm{SO}_{4}$. After filtration and removal of solvent under reduced pressure the residue was purified by column chromatography using silica gel (20\% EtOAc/hexane) to afford 3a (62.8 mg, 90\% yield).

Characterization DataMethyl (E)-3-(2-\{[1,2,3]Triazolo[1,5a]pyridin-3-yl\}phenyl)acrylate (3a)

Yield (62.8 mg, 90\% yield, semisolid), eluent $25 \%$ ethyl acetate/hexane. ${ }^{1} \mathrm{H}$ NMR $\left(600 \mathrm{MHz}, \mathrm{CDCl}_{3}\right): \delta=8.80(\mathrm{~d}, J=7.2 \mathrm{~Hz}, 1$ H), $7.89(\mathrm{~d}, J=16.0 \mathrm{~Hz}, 1 \mathrm{H}), 7.81(\mathrm{~d}, J=7.7 \mathrm{~Hz}, 1 \mathrm{H}), 7.66-7.64$ $(\mathrm{m}, 2 \mathrm{H}), 7.53(\mathrm{t}, J=7.4 \mathrm{~Hz}, 1 \mathrm{H}), 7.49(\mathrm{~d}, J=7.2 \mathrm{~Hz}, 1 \mathrm{H}), 7.31-$ $7.27(\mathrm{~m}, 1 \mathrm{H}), 7.06(\mathrm{t}, J=6.8 \mathrm{~Hz}, 1 \mathrm{H}), 6.51(\mathrm{~d}, J=16.0 \mathrm{~Hz}, 1 \mathrm{H})$, $3.73(\mathrm{~s}, 3 \mathrm{H}) .{ }^{13} \mathrm{C}$ NMR $\left(150 \mathrm{MHz}, \mathrm{CDCl}_{3}\right): \delta=167.1,143.2,136.6$, 133.4, 132.0, 131.2, 130.8, 130.1, 128.7, 127.1, 125.8, 125.5, 119.3, 118.1, 115.5, 51.6. ESI-HRMS: $m / z[\mathrm{M}+\mathrm{Na}]^{+}$calcd for $\mathrm{C}_{19} \mathrm{H}_{15} \mathrm{NONa}$ : 302.0900 ; found: 302.0898 .

Ethyl $\quad(E)-3-(2-\{[1,2,3]$ Triazolo[1,5-a]pyridin-3-yl $\}$ phenyl)acrylate ( $3 \mathbf{b})$

Yield $(52.8 \mathrm{mg}, 72 \%$ yield, semisolid), eluent $20 \%$ ethyl acetate/hexane. ${ }^{1} \mathrm{H}$ NMR $\left(600 \mathrm{MHz}, \mathrm{CDCl}_{3}\right): \delta=8.79(\mathrm{dd}, J=6.9,2.7$ $\mathrm{Hz}, 1 \mathrm{H}), 7.85(\mathrm{~d}, J=16.0 \mathrm{~Hz}, 1 \mathrm{H}), 7.80(\mathrm{~d}, J=7.7 \mathrm{~Hz}, 1 \mathrm{H}), 7.66$ (dd, $J=15.4,8.1 \mathrm{~Hz}, 2 \mathrm{H}), 7.52-7.49(\mathrm{~m}, 1 \mathrm{H}), 7.47(\mathrm{t}, J=7.4 \mathrm{~Hz}, 1$ H), $7.28(\mathrm{~d}, J=6.9 \mathrm{~Hz}, 1 \mathrm{H}), 7.03(\mathrm{t}, J=6.9 \mathrm{~Hz}, 1 \mathrm{H}), 6.49(\mathrm{~d}, J=$ $15.4 \mathrm{~Hz}, 1 \mathrm{H}), 4.19-4.15(\mathrm{~m}, 2 \mathrm{H}), 1.25(\mathrm{td}, J=6.9,1.9 \mathrm{~Hz}, 3 \mathrm{H})$. ${ }^{13} \mathrm{C} \mathrm{NMR}\left(150 \mathrm{MHz}, \mathrm{CDCl}_{3}\right): \delta=166.6,143.0,136.6,133.4,132.0$, 131.2, 130.8, 130.1, 128.7, 127.1, 125.8, 125.5, 119.8, 118.1, 115.4, 60.4, 14.2. ESI-HRMS: $m / z[\mathrm{M}+\mathrm{Na}]^{+}$calcd for $\mathrm{C}_{17} \mathrm{H}_{15} \mathrm{~N}_{3} \mathrm{O}_{2} \mathrm{Na}$ : 316.1056; found: 316.1056 .

Butyl (E)-3-(2-\{[1,2,3]Triazolo[1,5-a]pyridin-3-yl $\}$ phenyl)acrylate (3c)

Yield $(69.1 \mathrm{mg}, 86 \%$ yield, semisolid), eluent $20 \%$ ethyl acetate/hexane. ${ }^{1} \mathrm{H}$ NMR $\left(600 \mathrm{MHz}, \mathrm{CDCl}_{3}\right): \delta=8.79(\mathrm{~d}, J=7.1 \mathrm{~Hz}, 1$ H), 7.83-7.79 (m, $2 \mathrm{H}), 7.67(\mathrm{~d}, J=7.3 \mathrm{~Hz}, 1 \mathrm{H}), 7.63(\mathrm{~d}, J=8.9 \mathrm{~Hz}$, $1 \mathrm{H}), 7.53-7.450(\mathrm{~m}, 1 \mathrm{H}), 7.48(\mathrm{t}, J=7.5 \mathrm{~Hz}, 1 \mathrm{H}), 7.29-7.26(\mathrm{~m}$, $1 \mathrm{H}), 7.04(\mathrm{q}, J=7.1 \mathrm{~Hz}, 1 \mathrm{H}), 6.50(\mathrm{~d}, J=15.9 \mathrm{~Hz}, 1 \mathrm{H}), 4.13(\mathrm{t}, J=$ $6.5 \mathrm{~Hz}, 2 \mathrm{H}), 1.61-1.55(\mathrm{~m}, 2 \mathrm{H}), 1.34-1.24(\mathrm{~m}, 2 \mathrm{H}), 0.91-0.86$ $(\mathrm{m}, 3 \mathrm{H}) .{ }^{13} \mathrm{C}$ NMR $\left(150 \mathrm{MHz}, \mathrm{CDCl}_{3}\right): \delta=166.7,142.9,142.2$, 136.6, 133.4, 132.0, 131.2, 130.9, 130.1, 128.7, 128.1, 127.0, 125.8, 125.5, 120.7, 119.8, 118.1, 115.4, 64.3, 30.6, 19.1, 19.0, 13.7. GC-MS: $m / z$ [M] calcd for $\mathrm{C}_{19} \mathrm{H}_{19} \mathrm{~N}_{3} \mathrm{O}_{2}$ : 321.1477; found: 321.00.

Methyl (E)-3-(2-\{[1,2,3]Triazolo[1,5-a]pyridin-3-yl\}phenyl)2-methylacrylate (3d)

Yield (63.8 $\mathrm{mg}, 87 \%$ yield, semisolid), eluent $25 \%$ ethyl acetate/hexane. ${ }^{1} \mathrm{H}$ NMR $\left(600 \mathrm{MHz}, \mathrm{CDCl}_{3}\right): \delta=8.76(\mathrm{~d}, J=7.1 \mathrm{~Hz}, 1$ H), 7.71-7.70 (m, $1 \mathrm{H}), 7.63(\mathrm{~s}, 1 \mathrm{H}), 7.55(\mathrm{~d}, J=8.7 \mathrm{~Hz}, 1 \mathrm{H})$, 7.50-7.46 (m, 3 H), 7.25-7.22 (m, $1 \mathrm{H}), 7.01(\mathrm{t}, J=6.7 \mathrm{~Hz}, 1 \mathrm{H})$, $3.68(\mathrm{~s}, 3 \mathrm{H}), 2.06(\mathrm{~s}, 3 \mathrm{H}) .{ }^{13} \mathrm{C}$ NMR $\left(150 \mathrm{MHz}, \mathrm{CDCl}_{3}\right): \delta=167.1$, 166.7, 143.7, 143.6, 141.0, 134.8, 133.9, 130.8, 130.7, 130.5, $129.8,129.2,129.1,128.4,127.9,124.0,122.9,120.4,112.8$, 52.5, 29.8. GC-MS: $m / z$ [M] calcd for $\mathrm{C}_{17} \mathrm{H}_{15} \mathrm{~N}_{3} \mathrm{O}_{2}$ : 293.1164; found: 293.10 .

2-Methoxyethyl (E)-3-(2-\{[1,2,3]Triazolo[1,5-a]pyridin-3-yl\}phenyl)acrylate (3e)

Yield (65.5 mg, 81\% yield, semisolid), eluent $25 \%$ ethyl acetate/hexane. ${ }^{1} \mathrm{H}$ NMR $\left(600 \mathrm{MHz}, \mathrm{CDCl}_{3}\right): \delta=8.83(\mathrm{~d}, J=7.0 \mathrm{~Hz}, 1$ H), $7.84(\mathrm{~s}, 1 \mathrm{H}), 7.82-7.80(\mathrm{~m}, 2 \mathrm{H}), 7.65(\mathrm{~d}, J=8.5 \mathrm{~Hz}, 2 \mathrm{H}), 7.53$ $(\mathrm{dd}, J=8.2,1.8 \mathrm{~Hz}, 1 \mathrm{H}), 7.36-7.32(\mathrm{~m}, 1 \mathrm{H}), 7.10(\mathrm{t}, J=6.7 \mathrm{~Hz}, 1$ $\mathrm{H}), 6.60(\mathrm{~d}, J=15.9 \mathrm{~Hz}, 1 \mathrm{H}), 4.34-4.32(\mathrm{~m}, 2 \mathrm{H}), 3.65-3.63(\mathrm{~m}, 2$ $\mathrm{H}), 3.41(\mathrm{~s}, 3 \mathrm{H}) .{ }^{13} \mathrm{C}$ NMR $\left(150 \mathrm{MHz}, \mathrm{CDCl}_{3}\right): \delta=166.4,142.4$, 
135.7, 135.1, 135.0, 132.2, 130.3, 129.8, 127.2, 126.3, 125.7, 120.7, 118.0, 115.7, 70.5, 63.8, 59.1. GC-MS: $\mathrm{m} / z$ [M] calcd for $\mathrm{C}_{18} \mathrm{H}_{17} \mathrm{~N}_{3} \mathrm{O}_{3}$ : 323.1270 ; found: 323.00 .

2-Hydroxyethyl $\quad(E)-3-(2-\{[1,2,3]$ triazolo[1,5-a]pyridin-3yl\}phenyl)but-2-enoate (3f)

Yield (56.6 mg, 70\% yield, semisolid), eluent 50\% ethyl acetate/hexane. ${ }^{1} \mathrm{H} \mathrm{NMR}\left(600 \mathrm{MHz}, \mathrm{CDCl}_{3}\right): \delta=8.76(\mathrm{~s}, 1 \mathrm{H}), 7.76(\mathrm{~d}$, $J=41.3 \mathrm{~Hz}, 2 \mathrm{H}), 7.60(\mathrm{~s}, 1 \mathrm{H}), 7.51-7.48(\mathrm{~m}, 3 \mathrm{H}), 7.26(\mathrm{~s}, 2 \mathrm{H})$, $7.01(\mathrm{~s}, 1 \mathrm{H}), 4.24(\mathrm{~s}, 2 \mathrm{H}), 3.78(\mathrm{~s}, 2 \mathrm{H}), 2.07(\mathrm{~s}, 3 \mathrm{H}) .{ }^{13} \mathrm{C}$ NMR $\left(150 \mathrm{MHz}, \mathrm{CDCl}_{3}\right): \delta=168.7,139.2,137.2,134.7,131.8,130.8$, 130.2, 128.8, 128.5, 128.1, 125.6, 118.4, 115.5, 66.6, 61.4, 14.2. GC-MS: $m / z$ [M] calcd for $\mathrm{C}_{18} \mathrm{H}_{17} \mathrm{~N}_{3} \mathrm{O}_{3}: 323.1270$; found: 323.00 . 2,2,2-Trifluoroethyl (E)-3-(2-\{[1,2,3]Triazolo[1,5-a]pyridin3-yl\}phenyl)acrylate (3g)

Yield (78.0 mg, $90 \%$ yield, semisolid), eluent $10 \%$ ethyl acetate/hexane. ${ }^{1} \mathrm{H}$ NMR $\left(600 \mathrm{MHz}, \mathrm{CDCl}_{3}\right): \delta=8.80(\mathrm{~d}, J=7.1 \mathrm{~Hz}, 1$ H), $8.02(\mathrm{~d}, J=15.9 \mathrm{~Hz}, 1 \mathrm{H}), 7.83(\mathrm{~d}, J=7.9 \mathrm{~Hz}, 1 \mathrm{H}), 7.69$ (d, $J=$ $7.8 \mathrm{~Hz}, 1 \mathrm{H}), 7.65(\mathrm{~d}, J=8.9 \mathrm{~Hz}, 1 \mathrm{H}), 7.55(\mathrm{t}, J=7.7 \mathrm{~Hz}, 1 \mathrm{H}), 7.49$ $(\mathrm{d}, J=7.6 \mathrm{~Hz}, 1 \mathrm{H}), 7.31-7.28(\mathrm{~m}, 1 \mathrm{H}), 7.06(\mathrm{t}, J=6.8 \mathrm{~Hz}, 1 \mathrm{H})$, $6.56(\mathrm{~d}, J=16.0 \mathrm{~Hz}, 1 \mathrm{H}), 4.55(\mathrm{q}, J=8.2 \mathrm{~Hz}, 2 \mathrm{H}) .{ }^{13} \mathrm{C}$ NMR $(150$ $\left.\mathrm{MHz}, \mathrm{CDCl}_{3}\right): \delta=165.0,164.2,146.9,145.8,136.4,132.9,132.1$, $131.7,130.8,130.3,129.6,129.4,129.1,128.9,128.0,127.4$, $126.7,126.2,125.7,125.7,125.6,125.5,124.0,122.2,118.5$, 118.2, 118.0, 117.4, 115.6, 115.6, 60.7, 60.4, 60.2, 60.0. GC-MS: $\mathrm{m} / z$ [M] calcd for $\mathrm{C}_{17} \mathrm{H}_{12} \mathrm{~F}_{3} \mathrm{~N}_{3} \mathrm{O}_{2}: 347.0882$; found: 347.00 .

(E)-3-(2-\{[1,2,3]Triazolo[1,5-a]pyridin-3-yl\}phenyl)acrylonitrile (3h)

Yield (56.0 mg, 91\% yield, semisolid), eluent 30\% ethyl acetate/hexane. ${ }^{1} \mathrm{H}$ NMR $\left(600 \mathrm{MHz}, \mathrm{CDCl}_{3}\right): \delta=8.82(\mathrm{~d}, J=7.0 \mathrm{~Hz}, 1$ H), 7.77 (d, $J=16.8 \mathrm{~Hz}, 1 \mathrm{H}), 7.72(\mathrm{~d}, J=7.8 \mathrm{~Hz}, 1 \mathrm{H}), 7.69$ (d, $J=$ $8.7 \mathrm{~Hz}, 1 \mathrm{H}), 7.64(\mathrm{~d}, J=7.6 \mathrm{~Hz}, 1 \mathrm{H}), 7.57(\mathrm{t}, J=7.5 \mathrm{~Hz}, 1 \mathrm{H}), 7.50$ $(\mathrm{t}, J=7.5 \mathrm{~Hz}, 1 \mathrm{H}), 7.36-7.33(\mathrm{~m}, 1 \mathrm{H}), 7.09(\mathrm{t}, J=6.9 \mathrm{~Hz}, 1 \mathrm{H})$, $5.94(\mathrm{~d}, J=17.0 \mathrm{~Hz}, 1 \mathrm{H}) \cdot{ }^{13} \mathrm{C}$ NMR $\left(150 \mathrm{MHz}, \mathrm{CDCl}_{3}\right): \delta=149.3$, 136.1, 132.7, 132.0, 131.0, 130.8, 130.6, 130.6, 128.8, 126.5, 126.3, 125.6, 118.1, 117.7, 115.7, 97.5. GC-MS: $m / z$ [M] calcd for $\mathrm{C}_{15} \mathrm{H}_{10} \mathrm{~N}_{4}$ : 246.0905; found: 246.20 .

2-Ethylhexyl (E)-3-(2-\{[1,2,3]Triazolo[1,5-a]pyridin-3yl)phenyl)acrylate (3i)

Yield (60.6 mg, 67\% yield, yellow liquid), eluent $15 \%$ ethyl acetate/hexane. ${ }^{1} \mathrm{H}$ NMR $\left(600 \mathrm{MHz}, \mathrm{CDCl}_{3}\right): \delta=8.80-8.79(\mathrm{~m}, 1 \mathrm{H})$, 7.78-7.72 (m, $2 \mathrm{H}), 7.62(\mathrm{t}, J=8.7 \mathrm{~Hz}, 2 \mathrm{H}), 7.49(\mathrm{dd}, J=8.1,2.3$ $\mathrm{Hz}, 1 \mathrm{H}), 7.31-7.29(\mathrm{~m}, 1 \mathrm{H}), 7.06(\mathrm{t}, J=6.5 \mathrm{~Hz}, 1 \mathrm{H}), 6.51(\mathrm{~d}, J=$ $16.1 \mathrm{~Hz}, 1 \mathrm{H}), 4.07(\mathrm{qd}, J=10.9,5.7 \mathrm{~Hz}, 2 \mathrm{H}), 1.56(\mathrm{t}, J=6.0 \mathrm{~Hz}, 1$ $\mathrm{H}), 1.31-1.24(\mathrm{~m}, 11 \mathrm{H}), 0.88-0.83(\mathrm{~m}, 8 \mathrm{H}) .{ }^{13} \mathrm{C}$ NMR $(150 \mathrm{MHz}$, $\left.\mathrm{CDCl}_{3}\right): \delta=166.6,141.6,135.7,135.1,134.9,132.2,132.1,130.2$, 129.8, 127.1, 126.2, 125.7, 121.1, 118.0, 115.6, 67.1, 38.8, 30.4, 29.0, 23.8, 23.0, 14.1, 11.1. GC-MS: $\mathrm{m} / \mathrm{z}[\mathrm{M}]$ calcd for $\mathrm{C}_{23} \mathrm{H}_{27} \mathrm{~N}_{3} \mathrm{O}_{2}$ : 377.2103; found: 377.30 .

Methyl (E)-3-(2-\{[1,2,3]Triazolo[1,5-a]pyridin-3-yl $\}-5-c h l o-$ rophenyl)acrylate (4a)

Yield (65.1 mg, 83\% yield, semisolid), eluent $25 \%$ ethyl acetate/hexane). ${ }^{1} \mathrm{H}$ NMR $\left(600 \mathrm{MHz}, \mathrm{CDCl}_{3}\right): \delta=8.80(\mathrm{~d}, J=7.0 \mathrm{~Hz}, 1$ H), 7.81-7.76 (m, $2 \mathrm{H}), 7.61-7.58(\mathrm{~m}, 2 \mathrm{H}), 7.49(\mathrm{~d}, J=8.1 \mathrm{~Hz}, 1$ $\mathrm{H}), 7.32-7.30(\mathrm{t}, J=6.0 \mathrm{~Hz}, 1 \mathrm{H}), 7.06(\mathrm{~d}, J=6.8 \mathrm{~Hz}, 1 \mathrm{H}), 6.50(\mathrm{~d}$, $J=16.1 \mathrm{~Hz}, 1 \mathrm{H}), 3.73(\mathrm{~s}, 3 \mathrm{H}) .{ }^{13} \mathrm{C} \operatorname{NMR}\left(150 \mathrm{MHz}, \mathrm{CDCl}_{3}\right): \delta=$ 166.7, 141.9, 135.5, 135.0, 134.8, 132.0, 130.1, 129.6, 127.1, $126.2,125.6,120.5,117.8,115.6,51.8$. GC-MS: $m / z$ [M] calcd for $\mathrm{C}_{16} \mathrm{H}_{12} \mathrm{ClN}_{3} \mathrm{O}_{2}$ : 313.0618; found: 313.00 .

Ethyl (E)-3-(2-\{[1,2,3]Triazolo[1,5-a]pyridin-3-yl\}-5-chlorophenyl)acrylate (4b)

Yield (61.3 mg, 75\% yield, semisolid), eluent 20\% ethyl acetate/hexane. ${ }^{1} \mathrm{H}$ NMR $\left(600 \mathrm{MHz}, \mathrm{CDCl}_{3}\right): \delta=8.77(\mathrm{~s}, 1 \mathrm{H}), 7.84-$ 7.79 (m, 2 H), 7.65 (s, 2 H), 7.50-7.46 (m, 2 H), 7.27 (s, 1 H), 7.02 $(\mathrm{s}, 1 \mathrm{H}), 6.49(\mathrm{~d}, J=15.7 \mathrm{~Hz}, 1 \mathrm{H}), 4.17(\mathrm{~s}, 2 \mathrm{H}), 1.24(\mathrm{~s}, 3 \mathrm{H}) .{ }^{13} \mathrm{C}$ $\operatorname{NMR}\left(150 \mathrm{MHz}, \mathrm{CDCl}_{3}\right): \delta=166.8,143.1,136.7,133.6,132.2$, $131.4,131.0,130.2,128.9,127.2,125.9,125.6,120.0,118.3$, 115.6, 60.5, 14.4. ESI-HRMS: $m / z[M+N a]^{+}$calcd for $\mathrm{C}_{17} \mathrm{H}_{14^{-}}$ $\mathrm{ClN}_{3} \mathrm{O}_{2} \mathrm{Na}$ : 350.0667 ; found: 350.0632 .

Butyl (E)-3-(2-\{[1,2,3]Triazolo[1,5-a]pyridin-3-yl\}-5-chlorophenyl)acrylate (4c)

Yield $(76.5 \mathrm{mg}, 86 \%$ yield, semisolid), eluent $20 \%$ ethyl acetate/hexane. ${ }^{1} \mathrm{H}$ NMR $\left(600 \mathrm{MHz}, \mathrm{CDCl}_{3}\right): \delta=8.80(\mathrm{~d}, J=7.1 \mathrm{~Hz}, 1$ H), $7.82(\mathrm{~d}, J=7.8 \mathrm{~Hz}, 1 \mathrm{H}), 7.68(\mathrm{~d}, J=7.3 \mathrm{~Hz}, 1 \mathrm{H}), 7.64(\mathrm{~d}, J=$ $8.9 \mathrm{~Hz}, 1 \mathrm{H}), 7.54(\mathrm{t}, J=7.4 \mathrm{~Hz}, 1 \mathrm{H}), 7.49(\mathrm{t}, J=7.5 \mathrm{~Hz}, 1 \mathrm{H}), 7.30-$ $7.27(\mathrm{~m}, 1 \mathrm{H}), 7.05(\mathrm{t}, J=6.8 \mathrm{~Hz}, 1 \mathrm{H}), 6.51(\mathrm{~d}, J=15.9 \mathrm{~Hz}, 1 \mathrm{H})$, $4.14(\mathrm{t}, J=6.5 \mathrm{~Hz}, 2 \mathrm{H}), 1.62-1.58(\mathrm{~m}, 2 \mathrm{H}), 1.36-1.33(\mathrm{~m}, 2 \mathrm{H})$, 0.92-0.88 (m, $3 \mathrm{H}) .{ }^{13} \mathrm{C}$ NMR $\left(150 \mathrm{MHz}, \mathrm{CDCl}_{3}\right): \delta=160.0,159.3$, $156.9,143.9,138.1,136.8,136.2,130.2,129.1,127.6,122.3$, $120.9,120.5,114.9,114.4,114.3,113.9,112.1,105.4,55.3,32.4$, 31.2, 30.3. GC-MS: $\mathrm{m} / \mathrm{z}[\mathrm{M}]$ calcd for $\mathrm{C}_{19} \mathrm{H}_{18} \mathrm{ClN}_{3} \mathrm{O}_{2}$ : 355.1058; found: 355.10 .

Methyl (E)-3-(2-\{[1,2,3]Triazolo[1,5-a]pyridin-3-yl\}-5-chlorophenyl)-2-methylacrylate (4d)

Yield (67.2 mg, $82 \%$ yield, semisolid), eluent $25 \%$ ethyl acetate/hexane. ${ }^{1} \mathrm{H}$ NMR $\left(600 \mathrm{MHz}, \mathrm{CDCl}_{3}\right): \delta=8.77(\mathrm{~d}, J=7.1 \mathrm{~Hz}, 1$ H), 7.72-7.70 (m, $1 \mathrm{H}), 7.64(\mathrm{~s}, 1 \mathrm{H}), 7.56(\mathrm{~d}, J=8.7 \mathrm{~Hz}, 1 \mathrm{H})$, $7.51-7.49(\mathrm{~m}, 1 \mathrm{H}), 7.48(\mathrm{~d}, J=3.3 \mathrm{~Hz}, 1 \mathrm{H}), 7.25(\mathrm{t}, J=7.32 \mathrm{~Hz}, 1$ $\mathrm{H}), 7.02(\mathrm{t}, J=6.7 \mathrm{~Hz}, 1 \mathrm{H}), 3.69(\mathrm{~s}, 3 \mathrm{H}), 2.07(\mathrm{~s}, 3 \mathrm{H}) .{ }^{13} \mathrm{C}$ NMR $\left(150 \mathrm{MHz}, \mathrm{CDCl}_{3}\right): \delta=160.0,134.8,131.2,129.9,129.0,128.5$, 126.5, 124.8, 124.2, 123.0, 121.7, 121.0, 115.0, 114.3, 109.2, 55.5, 29.7. GCMS: $m / z$ [M] calcd for $\mathrm{C}_{17} \mathrm{H}_{14} \mathrm{ClN}_{3} \mathrm{O}_{2}$ : 327.0775; found: 327.05 .

2-Methoxyethyl (E)-3-(2-\{[1,2,3]Triazolo[1,5-a]pyridin-3yl\}-5-chlorophenyl)acrylate (4e)

Yield (69.8 mg, 78\% yield, semisolid), eluent $25 \%$ ethyl acetate/hexane. ${ }^{1} \mathrm{H}$ NMR $\left(600 \mathrm{MHz}, \mathrm{CDCl}_{3}\right): \delta=8.79(\mathrm{~d}, J=7.0 \mathrm{~Hz}, 1$ $\mathrm{H}), 7.80-7.76(\mathrm{~m}, 2 \mathrm{H}), 7.60$ (d, $J=8.5 \mathrm{~Hz}, 2 \mathrm{H}), 7.49$ (dd, $J=8.2$, $1.8 \mathrm{~Hz}, 1 \mathrm{H}), 7.32-7.28(\mathrm{~m}, 1 \mathrm{H}), 7.06(\mathrm{t}, J=6.7 \mathrm{~Hz}, 1 \mathrm{H}), 6.56(\mathrm{~d}, J$ $=15.9 \mathrm{~Hz}, 1 \mathrm{H}), 4.30-4.28(\mathrm{~m}, 2 \mathrm{H}), 3.61-3.59(\mathrm{~m}, 2 \mathrm{H}), 3.37(\mathrm{~s}, 3$ H). ${ }^{13} \mathrm{C}$ NMR $\left(150 \mathrm{MHz}, \mathrm{CDCl}_{3}\right): \delta=166.2,142.2,135.5,134.9$, $134.8,132.0,130.2,129.7,127.1,126.2,125.6,120.5,117.8$, 115.6, 70.4, 63.7, 59.0. GC-MS: $m / z$ [M] calcd for $\mathrm{C}_{18} \mathrm{H}_{16} \mathrm{ClN}_{3} \mathrm{O}_{3}$ : 357.0880; found: 357.10 .

2-Hydroxyethyl (E)-3-(2-\{[1,2,3]Triazolo[1,5-a]pyridin-3-yl $\}-$ 5-chlorophenyl)but-2-enoate (4f)

Yield (58.1 mg, 65\% yield, semisolid), eluent $45 \%$ ethyl acetate/hexane). ${ }^{1} \mathrm{H}$ NMR $\left(600 \mathrm{MHz}, \mathrm{CDCl}_{3}\right): \delta=8.83(\mathrm{~d}, J=7.1 \mathrm{~Hz}, 1$ $\mathrm{H}), 7.84-7.80(\mathrm{~m}, 2 \mathrm{H}), 7.65(\mathrm{~d}, J=8.6 \mathrm{~Hz}, 2 \mathrm{H}), 7.53(\mathrm{~d}, J=8.3 \mathrm{~Hz}$, $1 \mathrm{H}), 7.36-7.32(\mathrm{~m}, 1 \mathrm{H}), 7.10(\mathrm{t}, J=6.8 \mathrm{~Hz}, 1 \mathrm{H}), 6.60(\mathrm{~d}, J=15.8$ $\mathrm{Hz}, 1 \mathrm{H}), 4.33(\mathrm{t}, J=4.7 \mathrm{~Hz}, 2 \mathrm{H}), 3.65(\mathrm{t}, J=4.7 \mathrm{~Hz}, 2 \mathrm{H}), 3.41(\mathrm{~s}, 3$ H). ${ }^{13} \mathrm{C}$ NMR $\left(150 \mathrm{MHz}, \mathrm{CDCl}_{3}\right): \delta=168.7,139.2,137.2,134.6$, $131.8,130.8,130.2,128.7,128.5,128.1,125.6,118.4,115.5$, 66.6, 61.4, 14.2. GC-MS: $\mathrm{m} / z[\mathrm{M}]$ calcd for $\mathrm{C}_{18} \mathrm{H}_{16} \mathrm{ClN}_{3} \mathrm{O}_{3}$ : 357.0880; found: 357.10 .

2,2,2-Trifluoroethyl (E)-3-(2-\{[1,2,3]Triazolo[1,5-a]pyridin3-yl\}-5-chlorophenyl)acrylate (4g)

Yield (84 mg, 88\% yield, semisolid), eluent 25\% ethyl acetate/hexane. ${ }^{1} \mathrm{H}$ NMR $\left(600 \mathrm{MHz}, \mathrm{CDCl}_{3}\right): \delta=8.71(\mathrm{~d}, J=7.2 \mathrm{~Hz}, 1$ $\mathrm{H}), 7.93(\mathrm{~d}, J=16.0 \mathrm{~Hz}, 1 \mathrm{H}), 7.75(\mathrm{~d}, J=8.0 \mathrm{~Hz}, 1 \mathrm{H}), 7.60(\mathrm{~d}, J=$ $8.0 \mathrm{~Hz}, 1 \mathrm{H}), 7.57(\mathrm{~d}, J=9.0 \mathrm{~Hz}, 1 \mathrm{H}), 7.48(\mathrm{t}, J=7.6 \mathrm{~Hz}, 1 \mathrm{H}), 7.42$ (q, $J=7.8,7.3 \mathrm{~Hz}, 1 \mathrm{H}), 6.97(\mathrm{t}, J=6.9 \mathrm{~Hz}, 1 \mathrm{H}), 6.47(\mathrm{~d}, J=16.0$ $\mathrm{Hz}, 1 \mathrm{H}), 4.46$ (q, $J=8.1 \mathrm{~Hz}, 2 \mathrm{H}) .{ }^{13} \mathrm{C} \mathrm{NMR}\left(150 \mathrm{MHz}, \mathrm{CDCl}_{3}\right): \delta=$ $167.0,166.6,143.6,143.5,140.9,134.7,133.8,130.7,130.6$, $130.4,129.7,129.1,129.0,128.3,127.8,123.9,122.8,120.3$, 112.7, 52.4, 29.7. GC-MS: $m / z[M]$ calcd for $\mathrm{C}_{17} \mathrm{H}_{11} \mathrm{ClF}_{3} \mathrm{~N}_{3} \mathrm{O}_{2}$ : 381.0492; found: 381.30 . 
(E)-3-(2-\{[1,2,3]Triazolo[1,5-a]pyridin-3-yl\}-5-chlorophenyl)acrylonitrile (4h).

Yield (60.4 mg, 86\% yield, semisolid), eluent $25 \%$ ethyl acetate/hexane. ${ }^{1} \mathrm{H}$ NMR $\left(600 \mathrm{MHz}, \mathrm{CDCl}_{3}\right): \delta=8.24(\mathrm{~d}, J=7.8 \mathrm{~Hz}, 1$ $\mathrm{H}), 8.02(\mathrm{~d}, J=6.7 \mathrm{~Hz}, 1 \mathrm{H}), 7.80(\mathrm{~d}, J=8.0 \mathrm{~Hz}, 1 \mathrm{H}), 7.72(\mathrm{~d}, J=$ $9.2 \mathrm{~Hz}, 1 \mathrm{H}), 7.48(\mathrm{t}, J=7.5 \mathrm{~Hz}, 2 \mathrm{H}), 7.39(\mathrm{t}, J=7.5 \mathrm{~Hz}, 1 \mathrm{H}), 7.20$ $(\mathrm{t}, J=7.9 \mathrm{~Hz}, 1 \mathrm{H}), 6.81(\mathrm{t}, J=6.7 \mathrm{~Hz}, 1 \mathrm{H}) .{ }^{13} \mathrm{C}$ NMR $(150 \mathrm{MHz}$, $\left.\mathrm{CDCl}_{3}\right): \delta=149.3,145.7,140.1,128.9,125.3,125.2,124.3,124.1$, 124.0, 122.4, 121.5, 118.2, 111.9. GC-MS: $\mathrm{m} / \mathrm{z}[\mathrm{M}]$ calcd for $\mathrm{C}_{15} \mathrm{H}_{9} \mathrm{ClN}_{4}$ : 280.0516; found: 280.0 .

\section{Phenyl(pyridin-2-yl)methanone (5) ${ }^{\mathbf{1 1}}$}

Yield (14.0 mg, $31 \%$ yield, white solid), $\left.\mathrm{mp} 42-44{ }^{\circ} \mathrm{C}\right), 5 \%$ ethyl acetate/hexane. ${ }^{1} \mathrm{H}$ NMR $\left(600 \mathrm{MHz}, \mathrm{CDCl}_{3}\right): \delta=8.71(\mathrm{~d}, J=4.3$ $\mathrm{Hz}, 1 \mathrm{H}), 8.07(\mathrm{~d}, J=7.7 \mathrm{~Hz}, 2 \mathrm{H}), 8.03(\mathrm{~d}, J=7.8 \mathrm{~Hz}, 1 \mathrm{H}), 7.88(\mathrm{t}$, $J=7.7 \mathrm{~Hz}, 1 \mathrm{H}), 7.58(\mathrm{t}, J=7.3 \mathrm{~Hz}, 1 \mathrm{H}), 7.47(\mathrm{dd}, J=14.2,6.9 \mathrm{~Hz}$, $3 \mathrm{H}) .{ }^{13} \mathrm{C}$ NMR $\left(150 \mathrm{MHz}, \mathrm{CDCl}_{3}\right): \delta=193.7,154.9,148.4,136.9$, 136.1, 132.7, 130.8, 128.0, 126.0, 124.4.

(11) Joshi, A.; Kumar, R.; Semwal, R.; Rawat, D.; Adimurthy, S. Green Chem. 2019, 21, 962. 